\title{
Extending classical multirate signal processing theory to graphs
}

\section{Oguzhan Teke, Palghat P. Vaidyanathan}

Oguzhan Teke, Palghat P. Vaidyanathan, "Extending classical multirate signal processing theory to graphs," Proc. SPIE 10394, Wavelets and Sparsity XVII, 103941R (24 August 2017); doi: 10.1117/12.2272362

SPIE Event: SPIE Optical Engineering + Applications, 2017, San Diego, California, United States 


\title{
Extending Classical Multirate Signal Processing Theory to Graphs
}

\author{
Oguzhan Teke and Palghat P. Vaidyanathan \\ California Institute of Technology, Pasadena, CA, USA
}

\begin{abstract}
A variety of different areas consider signals that are defined over graphs. Motivated by the advancements in graph signal processing, this study first reviews some of the recent results on the extension of classical multirate signal processing to graphs. In these results, graphs are allowed to have directed edges. The possibly non-symmetric adjacency matrix $\boldsymbol{A}$ is treated as the graph operator. These results investigate the fundamental concepts for multirate processing of graph signals such as noble identities, aliasing, and perfect reconstruction (PR). It is shown that unless the graph satisfies some conditions, these concepts cannot be extended to graph signals in a simple manner. A structure called $M$-Block cyclic structure is shown to be sufficient to generalize the results for bipartite graphs on two-channels to $M$-channel filter banks. Many classical multirate ideas can be extended to graphs due to the unique eigenstructure of $M$-Block cyclic graphs. For example, the PR condition for filter banks on these graphs is identical to PR in classical theory, which allows the use of well-known filter bank design techniques. In order to utilize these results, the adjacency matrix of an $M$-Block cyclic graph should be given in the correct permutation. In the final part, this study proposes a spectral technique to identify the hidden $M$-Block cyclic structure from a graph with noisy edges whose adjacency matrix is given under a random permutation. Numerical simulation results show that the technique can recover the underlying $M$-Block structure in the presence of random addition and deletion of the edges.
\end{abstract}

Keywords: Multirate processing, graph signals, block-cyclic graphs, spectral approximation.

\section{INTRODUCTION}

The recent technological era brings signals that cannot be represented via classical time series. In most cases these high-dimension signals have irregular patterns. Graphical models are useful to model the inherent dependency structure of such signals where a graph signal is considered as a set of data points indexed according to vertices of the graph. This is a very generic model that can be used in variety of different contexts such as social and economic networks, ${ }^{1}$ biological networks ${ }^{2}$ and others. ${ }^{3}$

In order to manipulate these graph signals, there are studies that generalize the classical signal processing theory to graph signals. In the recent literature, there are two new approaches. The first one assumes an undirected graph structure with non-negative edge values and focuses on the graph Laplacian, ${ }^{4}$ which is defined for undirected graphs as $\mathcal{L}=\mathcal{D}-\boldsymbol{A}$ where $\mathcal{D}$ is the diagonal degree matrix and $\boldsymbol{A} \in \mathcal{C}^{N \times N}$ is the adjacency matrix of the graph. This construction is based on spectral graph theory ${ }^{5}$ and reveals very interesting results. In the second study, the adjacency matrix of the graph, $\boldsymbol{A}$, is considered as the unit-shift operator on the graph signal. $^{6-8}$ In this construction, the adjacency matrix is allowed to be asymmetric and can also have complex values as the edge weights. Therefore, more general classes of graphs can be considered under this theory. Many fundamental concepts in linear system theory have been generalized to the case of graph signals. ${ }^{6,7,9-12}$

Multirate systems and filter banks find many applications in signal processing theory and implementations. ${ }^{13,14}$ Recently, 2-channel filter banks for graph signals were developed. ${ }^{15}$ It is possible to obtain $M$-channel filter banks for even $M$ via iteratively using 2-channel decomposition and oversampling the graph. ${ }^{16}$ Both of these approaches depend on the graph Laplacian, hence they are restricted to undirected graphs with non-negative edge values.

Contact e-mails: oteke@caltech.edu, ppvnath@systems.caltech.edu. This work was supported in parts by the ONR grant N00014-15-1-2118, the NSF grant CCF-1712633, and the Electrical Engineering Carver Mead Research Seed Fund of the California Institute of Technology.

Wavelets and Sparsity XVII, edited by Yue M. Lu, Dimitri Van De Ville, Manos Papadakis, Proc. of SPIE Vol. 10394, 103941R · (C) 2017 SPIE · CCC code: 0277-786X/17/\$18 · doi: 10.1117/12.2272362 
The goal of this study is twofold. In Sections 2-4, we will first review some recent results on the multirate processing of graph signals, ${ }^{17,18}$ which consider the concepts such as decimation, expansion, noble identities and filter banks for an arbitrary rate $M$. In general these concepts do not extend to graphs in a straightforward manner. A class of graphs called $M$-block cyclic graphs plays an important role in order to relate the classical theory to graphs. However, not all graphs are $M$-Block cyclic. Therefore, these results are applicable to a subset of graphs. In the second part of this study (Section 5) we consider the problem of $M$-Block cyclic approximation of a given graph. We have observed that the eigenvector structure of $M$-Block cyclic graphs is robust to random insertion and deletion of edges. Hence, it is possible to recover the underlying structure even if the given graph is not exactly $M$-Block cyclic.

\subsection{Notation \& Preliminaries}

Given a graph, $\boldsymbol{A}$ represents the adjacency matrix of the graph, whose $(i, j)^{t h}$ element denotes the weight of the edge from the $j^{t h}$ node to the $i^{t h}$ node. This definition is the reverse of the usual definition in graph theory, but it is common in graph signal processing. ${ }^{6,8}$ The size of the graph and the length of the signal on the graph will be denoted by $N$, and $M$ denotes the decimation ratio or the number of filters in a graph filter bank, according to context. Given the following eigenvalue decomposition $\boldsymbol{A}=\boldsymbol{V} \boldsymbol{\Lambda} \boldsymbol{V}^{-1}$, the eigenvector matrix $\boldsymbol{V}$ will be referred to as graph Fourier basis. ${ }^{8}$ A graph filter $\boldsymbol{H}$ is said to be polynomial if it can be written as a polynomial in the adjacency matrix $\boldsymbol{A}$ :

$$
H(\boldsymbol{A})=\sum_{k=0}^{N-1} h_{k} \boldsymbol{A}^{k},
$$

for some set of possibly complex coefficients $h_{k}$ 's. For a matrix $\boldsymbol{A}$, its conjugate transpose will be denoted as $\boldsymbol{A}^{H}$, and its transpose will be denoted as $\boldsymbol{A}^{T}$. The Kronecker product will be denoted with $\otimes$.

\section{FUNDAMENTALS}

\subsection{Downsampling and Upsampling Operations}

One of the most essential building blocks for multirate signal processing on graphs is the decimation operation. ${ }^{13}$ This operator will be assumed to retain $N / M$ samples of the original graph signal $\boldsymbol{x}$, which has $N$ samples. It is assumed that $M$ is a divisor of $N$. Since the numbering convention for the graph vertices is arbitrary ${ }^{6}$ the decimator retains the first $N / M$ samples of the graph signal $\boldsymbol{x}$ without loss of any generality. This assumption requires a proper permutation of the nodes, which may not be available in general. Later in Section 5 we will discuss how to handle this issue. The graph decimation operator is defined as follows:

$$
\boldsymbol{D}=\left[\begin{array}{llll}
\boldsymbol{I}_{N / M} & \mathbf{0}_{N / M} & \cdots & \mathbf{0}_{N / M}
\end{array}\right] \in \mathcal{C}^{(N / M) \times N},
$$

where $\boldsymbol{I}_{N / M}$ is the identity matrix of size $N / M$. The decimated graph signal is $\boldsymbol{D} \boldsymbol{x}$. We refer to $\boldsymbol{D}$ as the canonical decimator with decimation ratio $M$. This is a mapping from $N$ to $N / M$ dimensional space.

Next, the upsampling operation $\boldsymbol{U} \in \mathcal{C}^{N \times(N / M)}$ is a mapping from $N / M$ dimensional complex space to $N$ dimensional complex space. Once the downsampling is defined, the upsampling operator cannot be chosen arbitrarily, they should be consistent with each other. In general, downsample-then-upsample is a lossy operation. Contrary to that, upsample-then-downsample operator is expected to be equal to identity. That is to say

$$
\boldsymbol{D} \boldsymbol{U}=\boldsymbol{I}_{N / M} .
$$

For a given $\boldsymbol{D}$, the right inverse $\boldsymbol{U}$ is not unique. When the minimum norm solution is considered, the upsampling operator can be found as $\boldsymbol{U}=\boldsymbol{D}^{H}\left(\boldsymbol{D} \boldsymbol{D}^{H}\right)^{-1}$, assuming that $\boldsymbol{D}$ has full row rank. This result reduces to

$$
\boldsymbol{U}=\boldsymbol{D}^{T}=\left[\begin{array}{c}
\boldsymbol{I}_{N / M} \\
\mathbf{0}_{N / M} \\
\vdots \\
\mathbf{0}_{N / M}
\end{array}\right] \in \mathcal{C}^{N \times(N / M)}
$$


for the decimator operator defined in (2). Hence, the corresponding uniform upsampler with expansion ratio $M$ is defined by the matrix $\boldsymbol{D}^{T}$. This operation merely inserts blocks of zeros, analogous to conventional expanders. ${ }^{13,14}$

In the following, results will be based on the simple canonical $\boldsymbol{D}$ defined in (2). More generally, decimator can be selected as an arbitrary $(N / M) \times N$ matrix with full row-rank. Such a definition provides an extension to the results reviewed here. ${ }^{18}$

\subsection{Noble Identities}

In classical signal processing, we have the first noble identity described in Figure 1(a) and the second noble identity described in Figure 1(b), where $H(z)$ denotes the $z$-transform of an LTI filter. For graph signals, analogous results should be obtained for the development of multirate systems. The analogous identities would be as in Figure 1(c) and 1(d), respectively. In classical signal processing the unit delay $z^{-1}$ has the same meaning for both original and decimated signals. But for graph signals, the elementary shift operator should match the size of the signal. Due to the mismatch in the size of the lower rate (of length $N / M$ ) and the higher rate (of length $N$ ) signals, an adjusted shift operators for these signals should be defined. For this reason, $\overline{\boldsymbol{A}}$ and $\tilde{\boldsymbol{A}}$ will be used to denote the adjusted shift operators for the first and the second noble identities, respectively.

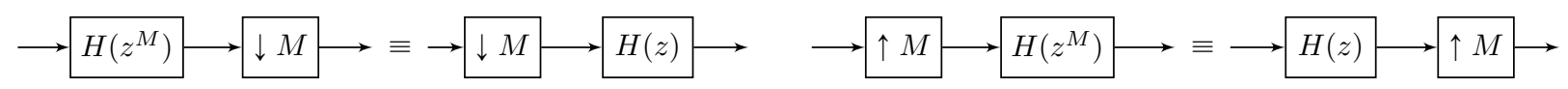

(a)

(b)

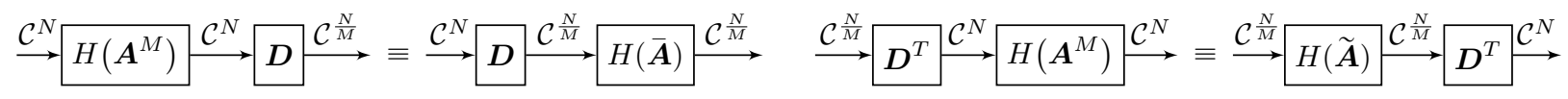

(c)

(d)

Figure 1. (a) The first noble identity, (b) the second noble identity in the classical signal processing. (c) The first noble identity, (d) the second noble identity in the case of graph signals on the adjacency matrix $\boldsymbol{A}$.

In general, conditions on the graph under which the noble identities exist are different for both noble identities. ${ }^{17}$ Furthermore, the adjusted shift operators cannot be assumed to be the same for both noble identities. In multirate theory, the two noble identities are used in conjunction, hence the graph should satisfy the identities in Figures 1(c) and 1(d) simultaneously. The following theorem, due to Teke and Vaidyanathan, precisely describes the condition on the graph and the adjusted shift operator such that both the noble identities exist. ${ }^{17}$

Theorem 1 (The noble identities). For a graph $\boldsymbol{A}$, the two noble identities

$$
\begin{gathered}
\boldsymbol{D} H\left(\boldsymbol{A}^{M}\right)=H(\overline{\boldsymbol{A}}) \boldsymbol{D}, \\
H\left(\boldsymbol{A}^{M}\right) \boldsymbol{D}^{T}=\boldsymbol{D}^{T} H(\overline{\boldsymbol{A}}),
\end{gathered}
$$

are simultaneously satisfied for all polynomial filters $H(\cdot)$ if and only if the following two equations are satisfied: $\boldsymbol{A}^{M}$ has the form

$$
\boldsymbol{A}^{M}=\left[\begin{array}{cc}
\left(\boldsymbol{A}^{M}\right)_{1,1} & \mathbf{0} \\
\mathbf{0} & \left(\boldsymbol{A}^{M}\right)_{2,2}
\end{array}\right]
$$

and

$$
\overline{\boldsymbol{A}}=\boldsymbol{D} \boldsymbol{A}^{M} \boldsymbol{D}^{T} \in \mathcal{C}^{(N / M) \times(N / M)}
$$

where $\left(\boldsymbol{A}^{M}\right)_{1,1} \in \mathcal{C}^{(N / M) \times(N / M)}$.

Due to conditions provided by Theorem 1, it is easy to conclude that noble identities do not exist for arbitrary graphs. Furthermore, the conditions in the theorem are both necessary and sufficient. Hence, it fully characterizes the graphs for which noble identities are valid. In the following section a specific graph structure on which the noble identities exist will be considered. Furthermore, this graph structure will be shown to be useful in order to extend classical multirate theory to graphs. 


\section{3. $M$-BLOCK CYCLIC GRAPHS}

The two-channel filter bank theory has been extended to undirected bipartite graphs by Narang and Ortega. ${ }^{15}$ Contrary to intuition, the two-channel filter bank results on bipartite graphs do not extend to $M$-channel filter banks on $M$-partite graphs. ${ }^{17,18}$ With more restrictive conditions on the graph, it is possible to generalize the classical multirate theory to graph signals for arbitrary $M$. For this purpose the following graph structure is considered.

Definition 1 ( $M$-Block CYCLIC GRAPHS). A graph is said to be $M$-Block cyclic if the adjacency matrix of the graph has the following form:

$$
\boldsymbol{A}=\left[\begin{array}{cccccc}
\mathbf{0} & \mathbf{0} & \mathbf{0} & \cdots & \mathbf{0} & \boldsymbol{A}_{M} \\
\boldsymbol{A}_{1} & \mathbf{0} & \mathbf{0} & \cdots & \mathbf{0} & \mathbf{0} \\
\mathbf{0} & \boldsymbol{A}_{2} & \mathbf{0} & \cdots & \mathbf{0} & \mathbf{0} \\
\mathbf{0} & \mathbf{0} & \boldsymbol{A}_{3} & \ddots & \vdots & \vdots \\
\vdots & \vdots & \vdots & \ddots & \mathbf{0} & \mathbf{0} \\
\mathbf{0} & \mathbf{0} & \mathbf{0} & \cdots & \boldsymbol{A}_{M-1} & \mathbf{0}
\end{array}\right] \in \mathcal{M}^{N}
$$

where each $\boldsymbol{A}_{j}$ has arbitrary but appropriate sizes. Furthermore, such a graph is said to be balanced $M$-Block cyclic, when $\boldsymbol{A}_{j}$ 's have the same size, that is $\boldsymbol{A}_{j} \in \mathcal{C}^{(N / M) \times(N / M)}$. In this case, we can write the adjacency matrix as:

$$
(\boldsymbol{A})_{i, j}=\boldsymbol{A}_{j} \delta(j-i+1),
$$

where $(\cdot)_{i, j}$ denotes the $(i, j)^{\text {th }}$ block of the adjacency matrix and $\delta(\cdot)$ is the M-periodic discrete Dirac function, that is $\delta(M j)=1$ for all integer $j$ and zero otherwise.

In the rest of the paper, the focus will be on the balanced $M$-Block cyclic graphs. For the visual representation of $M$-Block cyclic graphs, see Figure 3(a) for a balanced 5-block cyclic graph of size 20. Also consider Figure 2 to see the relation between the $M$-Block cyclic matrices and the directed cyclic graph whose adjacency matrix is given as follows:

$$
\boldsymbol{C}_{N}=\left[\begin{array}{llll}
1 & & & 1 \\
& \ddots & \\
& & 1
\end{array}\right] \in \mathcal{M}^{N} .
$$

In the following, some properties of $M$-Block cyclic graphs will be presented. The adjacency matrix of an $M$-Block cyclic graph has many other properties as well. ${ }^{17,18}$

FACT 1. If a graph is $M$-Block cyclic, then it is $M$-partite, but not vice-versa.

FACT 2. A graph is 2-block cyclic if and only if it is bi-partite.

FACT 3. An $M$-Block cyclic graph is necessarily a directed graph for $M>2$, hence its adjacency matrix does not have any symmetry property in terms of edge weights.

FACT 4. A cyclic graph of size $N, \boldsymbol{C}_{N}$, is an $M$-Block cyclic graph for all $M$ that divides $N$. See Figure 2.

The special case of undirected bipartite graphs was considered extensively by Narang and Ortega for filter bank construction. ${ }^{15}$

\subsection{Eigen-properties of $M$-Block cyclic graphs}

$M$-block cyclic graphs have an important eigenvalue-eigenvector structure that plays a key role in the development of graph filter banks. ${ }^{17,18}$ This eigenvalue relation of block cyclic matrices has also been observed in earlier studies. ${ }^{19-22}$ This property is as follows: 


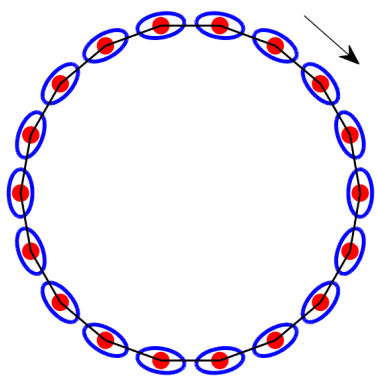

(a) $\boldsymbol{C}_{18}$ as 18 -block cyclic

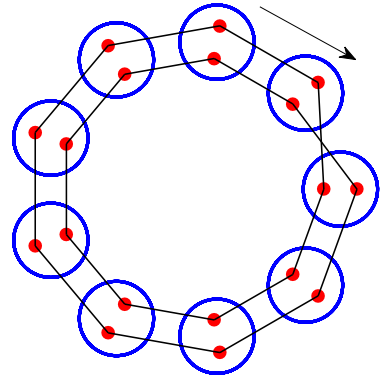

(b) $\boldsymbol{C}_{18}$ as 9 -block cyclic

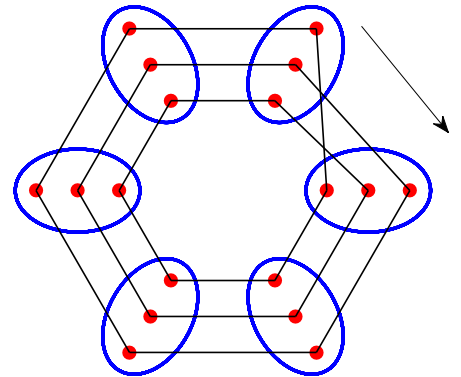

(c) $\boldsymbol{C}_{18}$ as 6-block cyclic

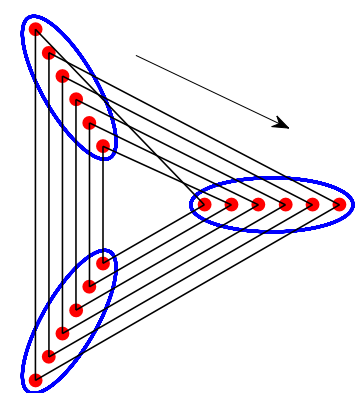

(d) $\boldsymbol{C}_{18}$ as 3-block cyclic

Figure 2. Under suitable permutation of the vertices, cyclic graph of size $N$ can be represented as an $M$-Block cyclic graph of size $N$ where $M$ divides $N$. Notice that cyclic graph of size $N$ is equivalent to $N$-Block cyclic graph of size $N$. All the edges are directed clock-wise as indicated by the arrow.

TheOREM 2 (EIGEN-FAMILIES OF $M$-BlOCK CYCliC GRAPHS). Eigenvalues and eigenvectors of the adjacency matrix of an $M$-Block cyclic graph come as families of size $M$. That is, if $(\lambda, \boldsymbol{v})$ is an eigenpair of $M$-Block cyclic graph, then $\left\{(\lambda, \boldsymbol{v}),(w \lambda, \boldsymbol{\Omega} \boldsymbol{v}),\left(w^{2} \lambda, \boldsymbol{\Omega}^{2} \boldsymbol{v}\right), \cdots\left(w^{M-1} \lambda, \boldsymbol{\Omega}^{M-1} \boldsymbol{v}\right)\right\}$ are all eigenpairs of the same graph, where

$$
\begin{gathered}
w=e^{-j 2 \pi / M}, \\
\boldsymbol{\Omega}=\operatorname{diag}\left(\left[1 w^{-1} w^{-2} \cdots w^{-(M-1)}\right]\right) \otimes \boldsymbol{I}_{N / M} .
\end{gathered}
$$

The weights of the edges in $\boldsymbol{A}$ affect the exact numerical values in the eigen-decomposition, but the eigenstructure (12), (13) is true for any $M$-block cyclic graph.

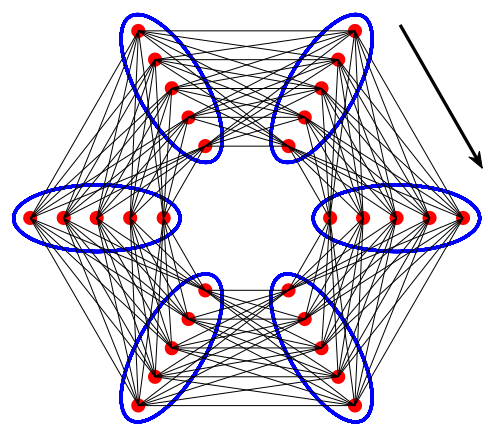

(a)

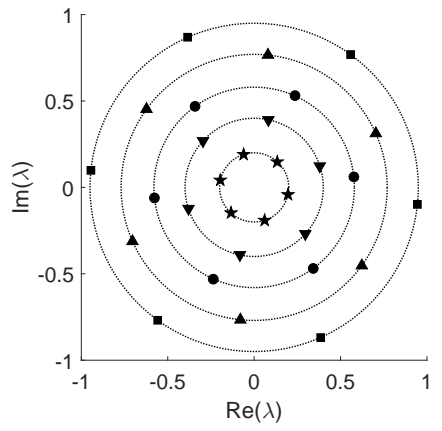

(b)

Figure 3. (a) 6-Block cyclic graph of size 30, (b) eigenvalues of the graph. Notice that all the edges are directed along with the clock-wise direction and they are allowed to have complex valued weights. As given in Theorem 2, eigenvalues of a balanced $M$-Block cyclic graph come as families of size $M$. Eigenvalues belonging to the same family are equally spaced on a circle in the complex plane.

Figure 3(b) visualizes the relation between the eigenvalues of an $M$-Block cyclic graph. There are $N / M$ concentric circles centered at the origin. Each circle has $M$ eigenvalues equispaced in angle. The circles need not have distinct radii.

Assuming that the adjacency matrix is diagonalizable, a double indexing scheme is used to represent the eigenvalues and the eigenvectors of $M$-Block cyclic graphs, since they come as families of size $M$. That is, the eigenpair $\left(\lambda_{i, j}, \boldsymbol{v}_{i, j}\right)$ will denote the $j^{t h}$ eigenpair of the $i^{t h}$ family, where $1 \leqslant i \leqslant N / M$ and $1 \leqslant j \leqslant M$. Using this indexing scheme, with the use of Theorem 2, the following relation exists between the eigenvalues and the 
eigenvectors:

$$
\begin{aligned}
& \lambda_{i, j+k}=w^{k} \lambda_{i, j}, \\
& \boldsymbol{v}_{i, j+k}=\boldsymbol{\Omega}^{k} \boldsymbol{v}_{i, j} .
\end{aligned}
$$

With this specific family structure of the eigenvalues of an $M$-Block cyclic graph, when the eigenvalue decomposition of the adjacency matrix is considered, $\boldsymbol{A}=\boldsymbol{V} \boldsymbol{\Lambda} \boldsymbol{V}^{-1}$, the eigenvalues and the eigenvectors are assumed to be ordered as follows:

$$
\boldsymbol{\Lambda}=\operatorname{diag}\left(\left[\lambda_{1,1} \cdots \lambda_{1, M} \cdots \lambda_{N / M, 1} \cdots \lambda_{N / M, M}\right]\right), \quad \boldsymbol{V}=\left[\boldsymbol{v}_{1,1} \cdots \boldsymbol{v}_{1, M} \cdots \boldsymbol{v}_{N / M, 1} \cdots \boldsymbol{v}_{N / M, M}\right] .
$$

\section{RATE- $M$ SYSTEMS}

It has been shown that $M$-Block cyclic matrices satisfy the noble identity condition given in Theorem 1 with the following adjusted shift matrix: ${ }^{17}$

$$
\overline{\boldsymbol{A}}=\boldsymbol{D} \boldsymbol{A}^{M} \boldsymbol{D}^{T}=\boldsymbol{A}_{M} \cdots \boldsymbol{A}_{1},
$$

where $\boldsymbol{A}_{i}$ 's are the partitions of the adjacency matrix given in (9). Furthermore, $M$-Block cyclic matrices are known to provide perfect reconstruction in the so-called delay-chain filter bank or lazy filter bank, which makes these graphs suitable for polyphase implementations. ${ }^{17}$ Some other uses of this graph structure will be presented next.

\subsection{Spectrum Folding}

In the development of classical multirate signal processing the concept of spectrum folding (or, aliasing) plays an important role. In order to study multirate processing of graph signals it is important to extend this phenomena to the case of graphs. For this purpose the downsample-then-upsample (DU) operation on graphs will be revisited. According to the canonical definition of decimator in (2) and the expander in (4), DU operator is given as follows:

$$
\boldsymbol{U} \boldsymbol{D}=\boldsymbol{D}^{T} \boldsymbol{D}=\left[\begin{array}{ll}
\boldsymbol{I} & \mathbf{0} \\
\mathbf{0} & \mathbf{0}
\end{array}\right]
$$

where $\boldsymbol{I}$ is an identity matrix of size $N / M$, and $\mathbf{0}$ are the blocks of zeros of appropriate sizes.

Given a graph signal $\boldsymbol{x}$ its downsampled-and-upsampled version can be written as $\boldsymbol{y}=\boldsymbol{D}^{T} \boldsymbol{D} \boldsymbol{x}$. Since DU operator replaces samples with zeros, it is a lossy operation and the erased samples of $\boldsymbol{x}$ cannot be reconstructed back from $\boldsymbol{y}$ in general. However, it is important to understand the relation between the spectral representations of these signals. For this purpose, let $\widehat{\boldsymbol{x}}$ and $\widehat{\boldsymbol{y}}$ denote the graph Fourier transforms of the signals $\boldsymbol{x}$ and $\boldsymbol{y}$, respectively. ${ }^{4,8}$ That is, $\widehat{\boldsymbol{x}}=\boldsymbol{V}^{-1} \boldsymbol{x}$ and $\widehat{\boldsymbol{y}}=\boldsymbol{V}^{-1} \boldsymbol{y}$. In the following, it will be assumed that the eigenvectors of the underlying graph have the restricted structure in (15). Eigenvalues, on the other hand, are not restricted, hence the structure in (14) is not necessary. Graphs that satisfy only the condition in (15) are referred to as $\boldsymbol{\Omega}$-graphs. ${ }^{18}$ To be consistent with the double indexing of the eigenvectors, the elements of $\widehat{\boldsymbol{x}}$ and $\hat{\boldsymbol{y}}$ will be indexed in accordance with the scheme in (16). That is to say,

$$
\widehat{\boldsymbol{x}}=\left[\widehat{x}_{1,1} \cdots \widehat{x}_{1, M} \cdots \widehat{x}_{N / M, 1} \cdots \widehat{x}_{N / M, M}\right]^{T}, \quad \hat{\boldsymbol{y}}=\left[\widehat{y}_{1,1} \cdots \widehat{y}_{1, M} \cdots \widehat{y}_{N / M, 1} \cdots \widehat{y}_{N / M, M}\right]^{T} .
$$

Similar to the approach presented for two-channel systems, ${ }^{15}$ it is shown that the spectral representations $\widehat{\boldsymbol{x}}$ and $\widehat{\boldsymbol{y}}$ are related as follows ${ }^{17}$ :

$$
\widehat{y}_{i, 1}=\widehat{y}_{i, 2}=\cdots=\widehat{y}_{i, M}=\frac{1}{M} \sum_{j=1}^{M} \widehat{x}_{i, j},
$$

for all $1 \leqslant i \leqslant N / M$. Thus the DU operation results in the phenomenon described by (20) in the frequency domain. This is similar to aliasing or spectral folding because multiple frequency components of the input overlap into the same frequency component of the output. This is similar to the effect of decimation in classical signal processing. ${ }^{13}$ From the folded spectrum (20) the original signal cannot be recovered in general, which is consistent with the fact that decimation is in general a information-lossy operation. 


\subsection{Filter Banks on $M$-Block Cyclic Graphs}

One of the topics of the multirate processing of graph signals is to develop $M$-channel filter banks as shown in Figure 4, where $H_{k}(\boldsymbol{A})$ and $F_{k}(\boldsymbol{A})$ are polynomials in the adjacency matrix $\boldsymbol{A}$, and $\boldsymbol{D}$ is the decimator defined in (2). The behavior of such graph filter banks differs from that of classical filter banks ${ }^{13,23}$ in many ways, the precise details depending on the eigenstructure of the adjacency matrix. ${ }^{18}$

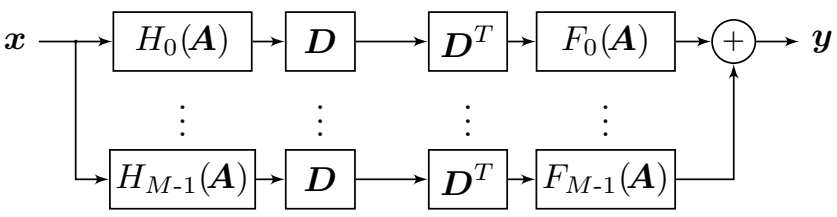

Figure 4. An $M$-channel maximally decimated filter bank on a graph with adjacency matrix $\boldsymbol{A}$. Here $H_{k}(\boldsymbol{A})$ and $F_{k}(\boldsymbol{A})$ are polynomials in $\boldsymbol{A}$ (so they are linear shift-invariant systems ${ }^{17}$ ). The decimation matrix $\boldsymbol{D}$ is as in $(2)$ with decimation ratio $M$. Overall response of the filter bank is denoted as $T(\boldsymbol{A})$, that is, $\boldsymbol{y}=T(\boldsymbol{A}) \boldsymbol{x}$.

When the adjacency matrix has unique eigenvalues one can design polynomial filters to construct and ideal filter bank. ${ }^{6,7,17}$ However, the order of such filters is $N$ in general, which is not practical to implement for graphs with large number of nodes. Furthermore, the filter coefficients are quite sensitive to the eigenvalues of the graph. Even a small change in the graph structure disturbs the perfect reconstruction property of such filter banks. Instead of ideal filter banks, which eliminate aliasing in each channel, it is possible to distribute the cancellation of aliasing among different channels. This allows a more general class of filters to analyze. While this idea is still not easy to develop for arbitrary graphs, the theory can be developed under some further assumptions on the graph, namely that $\boldsymbol{A}$ be $M$-Block cyclic. As long as the underlying graph is $M$-Block cyclic, it is possible to design polynomial filters that do not depend on the underlying eigenvalues of the adjacency matrix such that overall response of the filter bank is $T(\boldsymbol{A})=\boldsymbol{A}^{n}$ for some integer $n .{ }^{18}$ This result is as follows:

Theorem 3 (Perfect Reconstruction filter banks on $M$-Block Cyclic graphs). Consider the graph filter bank of Figure 4 and assume that the adjacency matrix of the graph is diagonalizable M-Block cyclic. With no further restrictions on the graph, the system has perfect reconstruction if and only if

$$
\sum_{k=0}^{M-1} F_{k}(\lambda) H_{k}\left(w^{l} \lambda\right)=M \lambda^{n} \delta(l),
$$

for some $n$, for all $\lambda \in \mathcal{C}$, and for all $l$ in $0 \leqslant l \leqslant M-1$, where $\delta(\cdot)$ is the discrete Dirac function.

It is quite interesting to observe that the condition (21) on the graph filters is the same as the PR condition in classical multirate theory. ${ }^{13}$ In order to design PR filter banks on an $M$-Block cyclic graph, any algorithm developed in the classical multirate theory ${ }^{13,14}$ can be used. Since $M$-Block cyclic graphs allow polyphase implementations for filter banks, ${ }^{18}$ more general conditions for alias-free filter banks is also obtained. ${ }^{18}$

\section{IDENTIFICATION OF THE $M$-BLOCK STRUCTURE FROM A NOISY PERMUTED VERSION}

Although many results from classical multirate theory can be extended to graphs with the help of $M$-Block cyclic graphs, the main limitation of these results is the restrictive nature of this condition. Unfortunately, most of the real world examples of graphs fail to satisfy this condition. The $M$-Block cyclic structure in (9) can be equivalently expressed in terms of the eigenvalue and eigenvector structure as given by Theorem 2 . Therefore, $M$-Block cyclic structure can be considered as constraints on both the eigenvalues and the eigenvectors. Using the idea of similarity transform it is possible to remove the constraint on the eigenvectors. ${ }^{18}$ Nevertheless, the condition on the eigenvalues is still necessary. Therefore, the results overviewed here are not applicable to all graphs. In some cases a given graph might be close to being an $M$-Block cyclic graph, but not exactly so. In this case it is necessary to identify the hidden $M$-Block cyclic structure in the graph, which can be considered as an approximation of a graph with an $M$-Block cyclic graph. 
Another subtle point with $M$-Block cyclic graphs is that Definition 1 implicitly assumes a proper labeling of the nodes such that the adjacency matrix can be written in the form of (9). However, the adjacency information of the graph might be provided without the required labeling of the nodes. In such a case the adjacency matrix cannot be written in the form of (9), hence the simple decimator and expander defined in (2) and (4) cannot be used directly.

Due to above mentioned points, this section considers an identification problem. Consider an unweighted $M$-Block cyclic graph where each of the nonzero blocks in $\boldsymbol{A}$ has the form $\mathbb{1}^{T}$ (i.e., these blocks are complete subgraphs). This is demonstrated in Figure 5(a). Instead of being given the exact adjacency matrix in Figure 5(a) (demonstrated for $M=5$ ), imagine we are given a modified version with the following modifications:

1. Some of the existing edges are removed randomly and independently with probability $q$ resulting in Figure 5(b).

2. Some new edges are inserted randomly and independently with probability $p$ resulting in Figure 5(c).

3. The $N$ nodes are randomly relabeled resulting in Figure 5(d).

Given the adjacency matrix corresponding to Figure 5(d), is it possible to recover the structure of the original $M$-Block cyclic graph? That is, invert the permutation that converted Figure 5(c) to Figure 5(d)? Once this is done, we can remove the randomly inserted edges to obtain Figure $5(\mathrm{~b})$, which is an $M$-Block cyclic graph with proper labeling of nodes. Of course we can never recover Figure 5(a) because of the randomly removed edges from the dark blocks. The main goal therefore is to identify the correct permutation of the labeling of the vertices so that we can go back from Figure 5(d) to Figure 5(c).

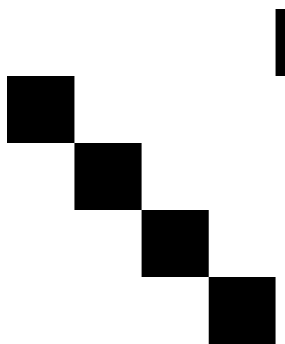

(a)
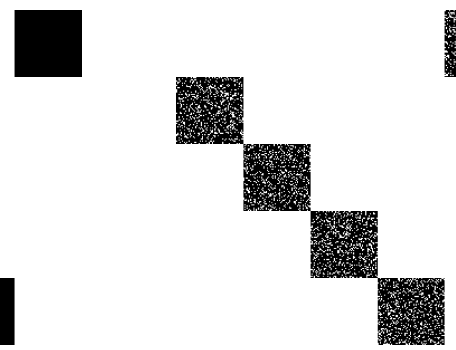

(b)

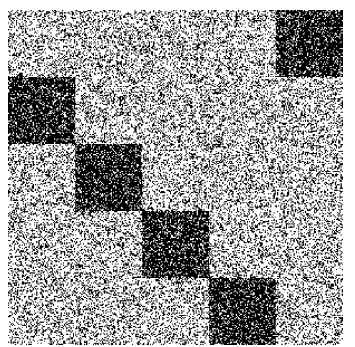

(c)

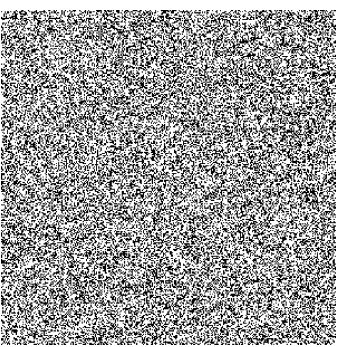

(d)

Figure 5. (a) The adjacency matrix of a 5-Block cyclic graph of size 300, (b) existing edges are removed with probability $q=0.2$, (c) noisy edges are added with probability $p=0.3$, (d) random re-labeling of the nodes.

In the case of $q=0$ and $p=0$ (no missing edges and noisy edges) a simple search can solve the labeling problem: start from a node. Outgoing edges reveals a block. Select a node from the identified block and repeat this procedure $M$ times. Since this simple technique implicitly assumes that the underlying graph is exactly $M$-Block cyclic, in the presence of missing and noisy edges such a procedure will fail. However, it is observed that the unique eigenvalue-eigenvector structure of $M$-Block cyclic matrices (given in Theorem 2) are robust to the missing and noisy edges, which can be used to identify the hidden blocks. In the following we will elaborate on this.

We will first consider the $M$-Block cyclic matrix whose adjacency matrix is visualized in Figure 5(a). Since all the edges are assumed to have unit weights, $\boldsymbol{A}$ can be written in the following close form:

$$
\boldsymbol{A}=\boldsymbol{C}_{M} \otimes\left(\mathbb{1}^{T}\right)
$$

where $\boldsymbol{C}_{M}$ is defined in (11) and $\mathbb{1} \in \mathcal{R}^{N / M}$ is the vector of all ones. Eigenvalues of the adjacency matrix $\boldsymbol{A}$ are then obtained as $\lambda(\boldsymbol{A})=\lambda\left(\boldsymbol{C}_{M}\right) \lambda\left(\mathbb{1} \mathbb{1}^{T}\right)$. Therefore $\lambda(\boldsymbol{A})=\left\{\frac{N}{M}, \omega \frac{N}{M}, \omega^{2} \frac{N}{M}, \cdots, \omega^{M-1} \frac{N}{M}, 0, \cdots, 0\right\}$ where $\omega$ is given in (12). The adjacency matrix $\boldsymbol{A}$ has $M$ non-zero eigenvalues equally-spaced on a circle with radius $N / M$. The remaining $N-M$ eigenvalues are all zeros. These eigenvalues are visualized in Figure 6 (a) for $M=5$ and $N=300$. When some of existing edges are removed, the adjacency matrix is no longer in the form of $(22)$. Hence, the eigenvalues of the matrix in Figure 5(b) are different than Figure 6(a). Since the removal of the edges does not disturb the $M$-Block cyclic property, all the eigenvalues still come as families of size $M$ due to 
Theorem 2. In addition, $M$ dominant eigenvalues continue to exist while the remaining ones are clustered around zero. Even though the magnitude of the dominant eigenvalues is a random variable (due to random removal of the edges), one can use Perron-Frobenius theorem for irreducible matrices ${ }^{24}$ to argue that the magnitude can be well approximated by (1-q) $N / M$. The eigenvalues of the matrix in Figure 5(b) are visualized in Figure 6(b). In this figure notice that $q$ is selected to be $q=0.2$.

In the presence of noisy edges $M$-Block cyclic structure no longer exist in the adjacency matrix. As a result, eigenvalues lose the family structure. However, it is interesting to observe that random addition of edges does not affect the structure of the dominant eigenvalues significantly. $M$ dominant eigenvalues (except for the one on the real line) continue to exist equally spaced on a circle (approximately) while the remaining ones are clustered around zero. The eigenvalues of the matrix in Figure 5(c) are visualized in Figure 6(c).

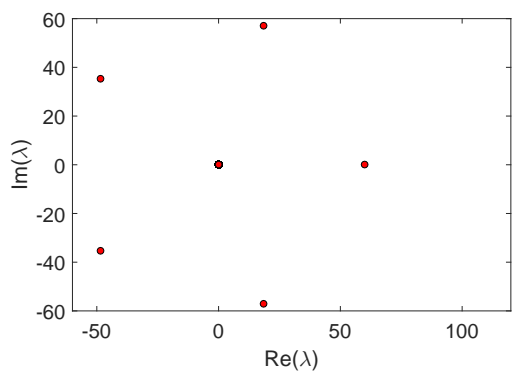

(a)

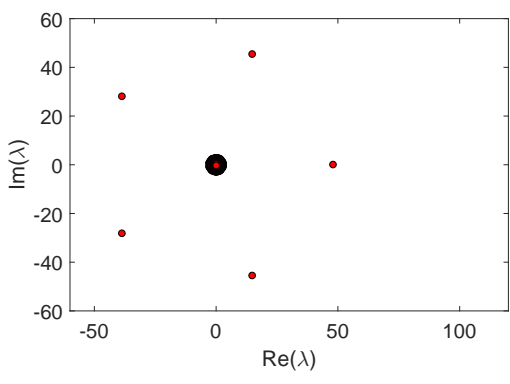

(b)

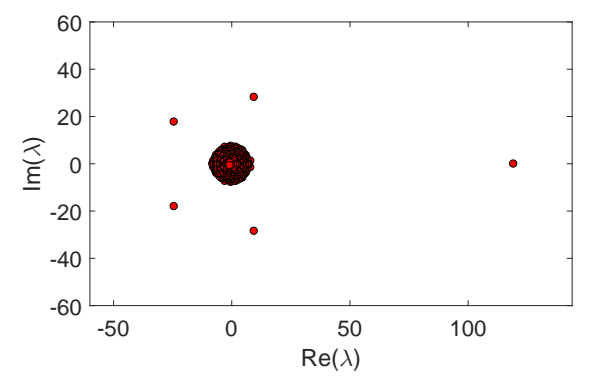

(c)

Figure 6. Eigenvalues of the matrices in (a) Figure 5(a), (b) Figure 5(b) and (c) Figures 5(c) and 5(d).

Due to stability of the eigenvalues one can hope to recover the underlying $M$-Block cyclic structure in the presence of noisy and missing edges. Based on this observation and inspired by the spectral graph coloring approach, ${ }^{25}$ we propose a 3 -step routine to identify the blocks of the underlying graph, which is outlined in Table 1.

Table 1. The proposed spectral technique for the identification of the $M$-Block cyclic structure

1. Given the adjacency matrix $\boldsymbol{A}$, compute its eigenvalue decomposition as $\boldsymbol{A}=\boldsymbol{V} \boldsymbol{\Lambda} \boldsymbol{V}^{-1}$ where eigenvalues are assumed to be ordered in the absolute sense, that is, $\left|\lambda_{1}\right| \geqslant \cdots\left|\lambda_{M}\right| \geqslant \cdots\left|\lambda_{N}\right|$. Let $\left\{\boldsymbol{v}_{1}, \cdots, \boldsymbol{v}_{M}\right\}$ be the eigenvectors that correspond to the largest (in the absolute sense) $M$ eigenvalues.

2. Stack the eigenvectors into a matrix as $\boldsymbol{S}=\left[\begin{array}{llll}\boldsymbol{v}_{1} & \boldsymbol{v}_{2} & \cdots & \boldsymbol{v}_{M}\end{array}\right]$, and compute the following $\boldsymbol{C}=\boldsymbol{S} \boldsymbol{S}^{H}$.

3. Cluster the rows of $\boldsymbol{C}$ into $M$ equal size partitions.

In this procedure the last step is not defined precisely. Various different techniques can be implemented. In our implementation we use a simple greedy technique to avoid the computational complexity: we start with the first row of the matrix $\boldsymbol{C}$. We find the indices of the elements of the vector with the largest $N / M$ values and assign these indices to a block. Then, we consider the smallest un-assigned index and repeat this procedure $M$ times. The rationale behind this greedy technique comes from the eigenvectors structure of the $M$-Block cyclic matrices (Theorem 2). In the case of $p=0$, the matrix $\boldsymbol{S}$ has the following structure:

$$
\boldsymbol{S}=\left[\begin{array}{ccccc}
\boldsymbol{u}_{1} & \boldsymbol{u}_{1} \omega^{0} & \boldsymbol{u}_{1} \omega^{0} & \ldots & \boldsymbol{u}_{1} \omega^{0} \\
\boldsymbol{u}_{2} & \boldsymbol{u}_{2} \omega^{-1} & \boldsymbol{u}_{2} \omega^{-2} & \ldots & \boldsymbol{u}_{2} \omega^{-(M-1)} \\
\vdots & \vdots & \vdots & \ddots & \vdots \\
\boldsymbol{u}_{M} & \boldsymbol{u}_{M} \omega^{-(M-1)} & \boldsymbol{u}_{M} \omega^{-2(M-1)} & \cdots & \boldsymbol{u}_{M} \omega^{-(M-1)(M-1)}
\end{array}\right], \quad \boldsymbol{C}=M\left[\begin{array}{ccc}
\boldsymbol{u}_{1} \boldsymbol{u}_{1}^{H} & & \\
& \ddots & \\
& & \boldsymbol{u}_{M} \boldsymbol{u}_{M}^{H}
\end{array}\right]
$$

where $\boldsymbol{u}_{i} \in \mathcal{R}^{N / M}$ are the partitions of the eigenvector $\boldsymbol{v}_{1}$. As a result, the matrix $\boldsymbol{C}$ has a block diagonal form as given in (23). In the case of $p>0$, the adjacency matrix is no longer $M$-Block cyclic. However, as suggested by 
Figure 6, the eigenvalue structure is not lost completely. Similarly, we have observed that the matrix $\boldsymbol{C}$ is "very close" to a block diagonal matrix. As a result, even a simple thresholding (finding the indices of the maximum values) is sufficient to identify the blocks of the hidden structure under a random re-labeling of the nodes.

When the proposed scheme is applied to the matrix in Figure 5(d), it finds a permutation (re-labeling of the nodes) that results in the adjacency matrix given in Figure 7 from which it is apparent that the algorithm successfully reveals the hidden $M$-Block cyclic structure. When carefully observed, one can realize that the matrices in Figures 5(d) and 7 are not equal to each other. There are two reasons for this mismatch: 1) The main purpose of the algorithm is to identify the blocks. The labeling of the nodes within a block is arbitrary. 2) Once the blocks are identified, numbering of the blocks (from 1 to $M$ ) is arbitrary. Nevertheless, from the multirate processing perspective Figure 5(c) and Figure 7 are equivalent to each other. The noisy edges can be removed, and previously discussed multirate techniques can be applied accordingly.

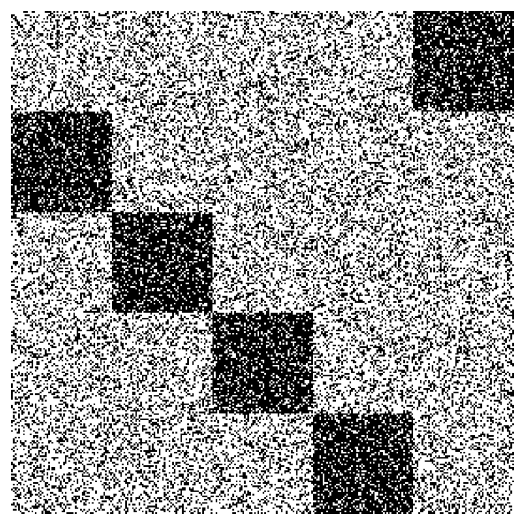

Figure 7. The algorithm proposed in Table 1 finds a permutation that converts Figure 5(d) to the above form.

We have tested the proposed scheme (Table 1) with various different values of $p$ and $q$. In order to evaluate the recovery performance we consider the fraction of the nodes that are labeled correctly. In the worst case scenario (the adjacency matrix does not have an $M$-Block cyclic structure) the routine randomly assigns the nodes to the blocks. Since there are $M$ blocks, the fraction can be at least $1 / M$ on the average. The recovery performance of the proposed technique is provided in Figure 8. The number of blocks is selected to be $M=5$, and the number of nodes are selected to be $N=100,300$ and 500 in Figures 8(a)-8(c), respectively.

It is important to note the trade-off between the parameters $p$ and $q$. The recovery performance of the proposed approach depends on the density of the edges in the regions. The average edge density between the consecutive blocks is 1- $q$ (since edges are removed with probability $q$ ), and the density in the remaining parts is $p$. Figure 5(c) visually describes the difference between the densities for the case of $q=0.2$ and $p=0.3$. In fact this difference keeps the structure on the eigenvalues intact. When $1-q \approx p$, the regions are no different from each other. Hence, the underlying blocks are not expected to be identified correctly. Figure 8 follows this expectation. Notice that the algorithm behaves poorly around the line $p+q=1$. In the vicinity of this region the fraction of correctly labeled nodes is $1 / M$, which corresponds to the random assignments of the nodes. Figures $8(\mathrm{a})-8(\mathrm{c})$ also suggest that larger number of nodes allows correct identification of the blocks for a larger set of values of $p$ and $q$.

As a final note, in the proposed algorithm the value of $M$ is assumed to be known, which may not be available in some cases. However, as Figure 6 suggests, even a visual analysis of the eigenvalues is enough to identify the value of $M$. If we were given only the Figure 6(c), then it would be straightforward to conclude that the eigenvalues belong to a 5-Block cyclic adjacency matrix. Hence, we can conclude that knowledge about $M$ is a very mild assumption.

\section{CONCLUSION}

In this paper, the fundamental blocks for multirate signal processing on graphs are reviewed. The canonical decimator is defined together with the corresponding expander. Necessary conditions on the graph for the 


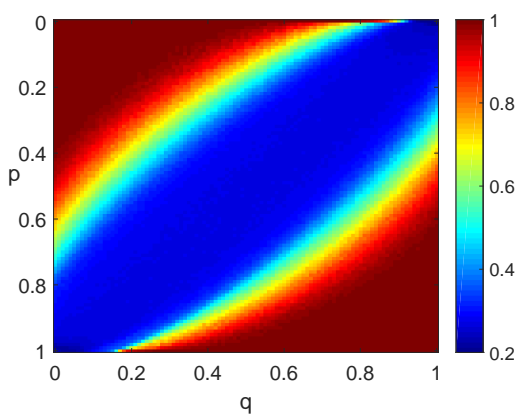

(a)

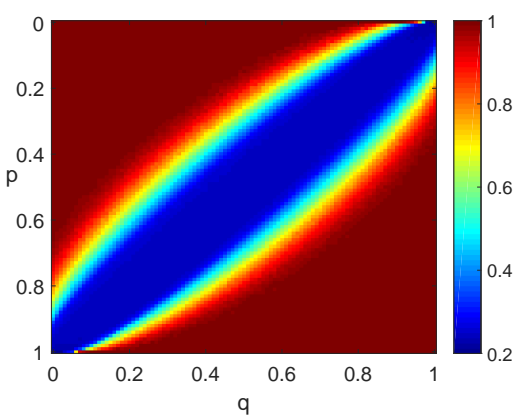

(b)

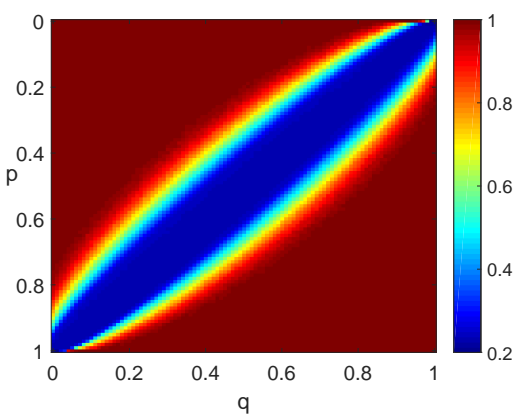

(c)

Figure 8. Recovery performance of the proposed technique for $M=5$. The fraction of the nodes that are labeled correctly is color coded. Number of nodes are (a) $N=100$, (b) $N=300$, (c) $N=500$. Fractions are averaged over 350 experiments.

existence of noble identities are shown, which implies that the extension of classical multirate signal processing theory to graphs requires certain mathematical restrictions on the adjacency matrix. In this regard so-called $M$ Block cyclic graphs are defined. Their eigenstructure is presented, and they are shown to be useful to relate the classical theory to graphs. In order to identify a hidden $M$-Block cyclic structure from the randomly permuted adjacency matrix with noisy edges, a spectral technique is proposed. This technique leverages the robustness of the eigenstructure of $M$-Block cyclic graphs. Numerical simulations show that the proposed technique is able to recover the underlying $M$-Block cyclic structure in the presence of random addition and deletion of the edges.

\section{ACKNOWLEDGMENTS}

The authors would like to thank Dr. Pierre Borgnat for the invitation to write this article.

\section{REFERENCES}

[1] Jackson, M. O., [Social and Economic Networks], Princeton University Press (2008).

[2] Weber, M. and Kube, S., "Robust perron cluster analysis for various applications in computational life science," in [Computational Life Sciences], 3695, 57-66, Springer Berlin Heidelberg (2005).

[3] Newman, M. E. J., [Networks: An Introduction], Oxford University Press (2010).

[4] Shuman, D., Narang, S., Frossard, P., Ortega, A., and Vandergheynst, P., "The emerging field of signal processing on graphs: Extending high-dimensional data analysis to networks and other irregular domains," IEEE Signal Processing Magazine 30, 83-98 (May 2013).

[5] Chung, F. R. K., [Spectral Graph Theory], AMS (1997).

[6] Sandryhaila, A. and Moura, J. M. F., "Discrete signal processing on graphs," IEEE Trans. Signal Process. 61, 1644-1656 (April 2013).

[7] Sandryhaila, A. and Moura, J. M. F., "Discrete signal processing on graphs: Frequency analysis," IEEE Trans. Signal Process. 62, 3042-3054 (June 2014).

[8] Sandryhaila, A. and Moura, J. M. F., "Big data analysis with signal processing on graphs: Representation and processing of massive data sets with irregular structure," IEEE Signal Processing Magazine 31, 80-90 (Sept 2014).

[9] Chen, S., Varma, R., Sandryhaila, A., and Kovacevic, J., "Discrete signal processing on graphs: Sampling theory," IEEE Trans. Signal Process. 63, 6510-6523 (Dec 2015).

[10] Agaskar, A. and Lu, Y. M., "A spectral graph uncertainty principle," IEEE Trans. on Inf. Theory 59, 4338-4356 (July 2013).

[11] Teke, O. and Vaidyanathan, P. P., "Sparse eigenvectors of graphs," in [Proc. Int. Conf. Acoust. Speech, Signal Process. (ICASSP)], 3904-3908 (March 2017).

[12] Teke, O. and Vaidyanathan, P. P., "Uncertainty principles and sparse eigenvectors of graphs," IEEE Trans. Signal Process. (to appear). 
[13] Vaidyanathan, P. P., [Multirate systems and filter banks], Englewood Cliffs, N.J. Prentice Hall (1993).

[14] Vaidyanathan, P. P., "Multirate digital filters, filter banks, polyphase networks, and applications: a tutorial," Proceedings of the IEEE 78, 56-93 (Jan 1990).

[15] Narang, S. and Ortega, A., "Perfect reconstruction two-channel wavelet filter banks for graph structured data," IEEE Trans. Signal Process. 60, 2786-2799 (June 2012).

[16] Tanaka, Y. and Sakiyama, A., "M-channel oversampled graph filter banks," IEEE Trans. Signal Process. 62, 3578-3590 (July 2014).

[17] Teke, O. and Vaidyanathan, P. P., "Extending classical multirate signal processing theory to graphs - Part I: Fundamentals," IEEE Trans. Signal Process. 65, 409-422 (Jan 2017).

[18] Teke, O. and Vaidyanathan, P. P., "Extending classical multirate signal processing theory to graphs - Part II: M-Channel filter banks," IEEE Trans. Signal Process. 65, 423-437 (Jan 2017).

[19] Watkins, D. S., "Product eigenvalue problems," SIAM Review 47(1), 3-40 (2005).

[20] Fiedler, M., [Special Matrices and Their Applications in Numerical Mathematics: Second Edition], Dover Publications (2008).

[21] Meyer, C. D., [Matrix analysis and applied linear algebra], Society for Industrial and Applied Mathematics (2000).

[22] Cvetkovic, D. M., Doob, M., and Sachs, H., [Spectra of Graphs: Theory and Application (Pure E3 Applied Mathematics)], Academic Press (1980).

[23] Vetterli, M. and Kovacevic, J., [Wavelets and Subband Coding], Prentice-Hall (1995).

[24] Horn, R. A. and Johnson, C. R., [Matrix Analysis], Cambridge University Press (1990).

[25] Aspvall, B. and Gilbert, J. R., "Graph coloring using eigenvalue decomposition," SIAM Journal on Algebraic Discrete Methods 5(4), 526-538 (1984). 